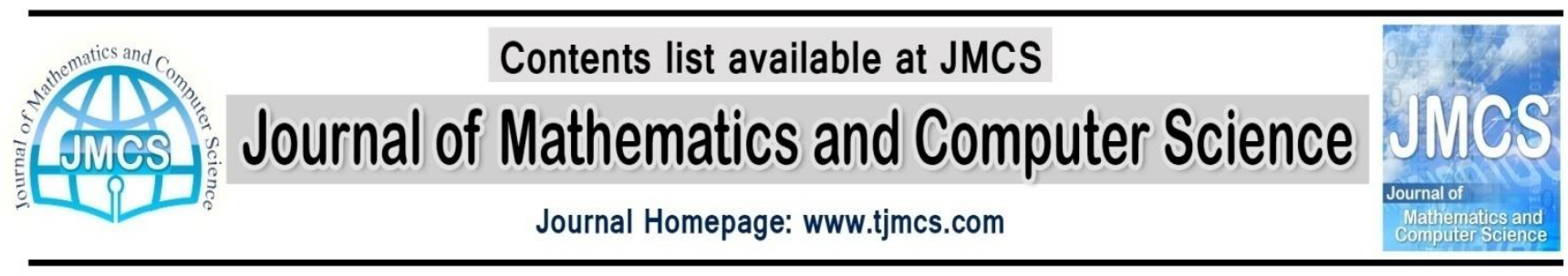

\title{
Multi-hop Fuzzy Routing for Wireless Sensor Network with Mobile Sink
}

\author{
Mohsen Nejadkheirallah \\ Allameh Mohaddes Noori Institute of Higher Education Noor, Iran \\ m.nejad@mohaddes.ac.ir \\ Mohammad Mahdi Tajari \\ Department computer sciense, Mashhad Branch, Islamic Azazd University, mashhad, Iran \\ mm.tajari@gmail.com \\ Reza Sookhtsaraei \\ Department of computer engineering and information technology, Payame Noor University, Iran \\ reza.sookhtsaraei@pnu.ac.ir \\ Ali Yousefzadeh \\ Allameh Mohaddes Noori Institute of Higher Education, Noor, Iran \\ yousefzadeh11@gmail.com
}

Article history:

Received August 2013

Accepted October 2013

Available online October 2013

\section{Abstract}

Using clustering methods in sensor networks improves their efficiency and management. Moreover, it brings with it the main parameter in sensor networks i.e. decrease in energy consumption. To transfer data to a sink in a network having many nodes, multi-hop methods have to be used. Also in many of the cases, there is a mobile sink (or user) in a network environment. In this paper, using fuzzy logic we present a routing method which divides the network into groups which are like clusters and has the ability of supporting the mobile sink. This routing method has been compared with other routing and clustering methods and the results show the efficiency of the mentioned method.

Keywords: wireless sensor network; multi-hop routing; fuzzy logic; mobile sink; multi-sink.

\section{Introduction}

ADhoc and sensor networks may have a large number of nodes deployed over wide areas, and nodes typically have limited battery and computational capabilities. They are often employed in environmental monitoring applications, which require their topology to be either fixed or slowly varying in a controllable manner, and their operational lifetime is of the order of weeks or months. 
However, this technology faces many challenges, one of the most challenging aspects Of WSNs is the energy constraint in the sensor nodes. The energy required by the sensor nodes is often provided by an exhaustible source of energy (e.g., batteries), which necessitates the usage of methods that can reduce the energy consumption in the network. In addition to electronic-based methods for adjusting the energy consumption of the sensors (e.g., better IC designs), many works have proposed better architecture designs to overcome this limit. Multi-hop packet transmission, in which the packet to be transmitted from a sensor is relayed by the nodes located at a shorter distance from the sink, is one of these ideas. Indeed, the multi-hop scheme is claimed to be more energy efficient compared to its equivalent single-hop network, since less transmission power is required for shorter distances. Despite many efforts to improve the energy efficiency of multi-hop-based WSNs [1]-[3], there is still uncertainty about its efficiency when more realistic models are taken into account. Large number node in WSN makes Clustering concept and clustering-algorithm widely use in this network. Cluster formation is typically based on the energy reserve of sensors and sensors proximity to the $\mathrm{CH}$ [4]. For instance, Low-Energy Adaptive Clustering Hierarchy (LEACH) [5], one of the first clustering protocols for WSN, is a distributed, proactive, dynamic algorithm that forms clusters of sensors based on the received signal strength and uses local $\mathrm{CHs}$ as routers to the sink. Weighted Clustering Algorithm (WCA) [6] is a reactive clustering algorithm where cluster election is based on the evaluation, for every sensor, of a score function called combined weight. This function is a weighted linear combination of the degree, the mobility level, the transmission power and the residual energy of the sensor. Hybrid Energy-Efficient Distributed Clustering (HEED) [10] is a distributed clustering protocol that uses a hybrid combination of the residual energy and the intra-cluster communication cost as attribute for cluster head selection. HEED ensures a uniform distribution of $\mathrm{CHs}$ across the network and adjusts the probability of $\mathrm{CH}$-selection to ensure inter- $\mathrm{CH}$ connectivity. In HEED, sensors compute a probability of becoming $\mathrm{CH}$, proportional to its residual energy and to a predetermined percentage of $\mathrm{CHs}$ and then, every sensor seeks the best $\mathrm{CH}$ to connect to. RCSDN [7] is a novel clustering algorithm a base on mean residual energy neighbor node, node come to $\mathrm{CH}$ candidate if residual energy node is most that mean residual energy neighbor node. Some of the clustering algorithms employ fuzzy logic to handle uncertainties in WSNs. Basically, fuzzy clustering Algorithms use fuzzy logic for blending different clustering parameters to elect cluster-heads.

In the fuzzy clustering Approach proposed by Gupta et al: the cluster-heads are elected at the base station. In every round, each sensor node forwards its clustering information to the base station. There are three fuzzy descriptors which are considered by the base station during cluster-head election. These fuzzy descriptors are node concentration, residual energy in each node and node centrality [8]. CHEF is a similar approach to that of Gupta et al: [8], but it performs cluster-head election in a distributed manner. Thus, the base station does not need to collect clustering information from all sensor nodes [9].

The introduction of mobility, either in the nodes or in the agents that collect data from them (that is, sinks), makes the design of networking protocols more challenging and complicated. In mobile-sink scenarios, frequently updating all nodes with a sink's current location leads to significant overheads. Recent literature suggests several alternative approaches. Directed diffusion [11] and its enhancements [12] route data based on data interests periodically broadcast by the sink but are incapable of accommodating high levels of network and sink dynamics. A scheme where each node builds a "grid" to route data to mobile sinks and thus incurs high overhead is proposed in [13]. The authors of [14] propose a set of algorithms that adaptively select a path that consists of a subset of nodes with high residual energy or a path with the least total power consumption. The energy-aware routing protocols proposed in [15] and [16] require power control, whereas this paper considers fixed transmission powers. Power-aware routing schemes that require the nodes to be aware of their geographic location are proposed in [17] and [18]. A probabilistic routing protocol is proposed in [19], where the probability of choosing a node as a forwarding node is inversely proportional to the aggregate load the node is carrying. 
The rest of the paper is organized as follows: In section 2, network model and energy consumption model is described. In section 3, our proposed fuzzy method is presented. . In section 4, the fuzzy module Design process is expressed. In section 5, multi-sink process is described. In section 6, mobilesink method is expressed. In section 7, the results of our Stimulation Analysis with our methods are also shown and conclusion of the study is in the last section.

\section{Network model and energy consumption model}

The network which we run FUMOR method on it has the following characteristics:

1) Sensor nodes and the base station are moveless after deployment.

2) The basic energy of nodes is different.

The model of energy consumption used in networks is the same as energy consumption model in LEACH model which has the following characteristics:

$$
\begin{cases}E_{T x}(n, d)=n\left(E_{\text {elec }}+\varepsilon_{f s} d^{2}\right) & d<d_{\mathrm{o}} \\ E_{T x}(n, d)=n\left(E_{\text {elec }}+\varepsilon_{m p} d^{4}\right) & d \geq d_{\mathrm{o}}\end{cases}
$$

Where, $\mathrm{n}$ is the number bit of the message and $\mathrm{d}$ is the distance, $E_{\text {elec }}$ is the energy dissipated per bit to run the transmitter or the receiver circuit, and $\varepsilon_{f s}, \varepsilon_{m p}$ is the energy dissipated per bit to run the transmit amplifier depending on the distance between the transmitter and receiver. If the distance is less than a threshold $d_{0}$, the free space (FS) model is used; otherwise, the multipath (MP) model is used. The energy consumption of the receiver is given by:

$$
E_{R x}(n)=n\left(E_{\text {elec }}\right)
$$

\section{The proposed plan}

In this section, the proposed algorithm will be examined and explained. The method suggested here, is a new one which enjoys the combination of cluster heads and gateways' role to transfer the data to the leader nodes and then to the sink through multi-hop process. In the proposed method which is called MHRMS (Multi-hop fuzzy routing for wireless sensor network with mobile sink), in addition to determining a fuzzy module in every node for qualifying it to be chosen as a $\mathrm{CH}$ (cluster-head or gateway), we want to examine the condition of data transfer to the sink after collecting data. Also through expanding the proposed plan, to add the capability of supporting the mobile-sink to the protocol.

To manage the sensor network, we have used a method similar to clustering method. The method of data transferring to sink is also a multi-hop one but in our method, the cluster heads are produced as a minimum spanning tree in the environment and choosing a node as a cluster head (or in other words a backbone to transfer the data to the sink) is done through the fuzzy logic. The nodes chosen as cluster head (gateway), does the task of receiving the data from their nodes and data aggregation operation. After this operation they send the data to their previous cluster head (gateway) which is closer to the sink in order for the data to be transferred to the sink.

In this plan, the nodes have been randomly spread in the environment and a sink has been put in the environment. The routing method is composed of some rounds each one which is implementation in two phases: The setup phase and the steady phase. In the setup phase the nodes create a path through themselves from the sink to the end of the network. In the steady phase, the nodes' data are transferred to the sink.

After the setup phase ends, every node in the environment takes some components such as: the variable of LEVEL which shows that to reach the sink from the current node, some steps (HOP) has to be traversed. As an example, a node having LEVEL; 6, must traverse 6 steps from this node to reach the sink. The next components are the high LEVEL gateway and the low LEVEL gateway. The former refers to the gateway node, its variable of LEVEL which is one unit less than the value of the current variable of LEVEL and the latter refers to the gateway node, its value of the variable of the LEVEL which is one unit more than the value of the current node's variable of LEVEL. 
The setup phase is as follows: in the setup phase, first, the sink sends a message called $\mathrm{CH}$-announce with a specified radius in which the parameter of LEVEL equals zero and it indicates that this nod i.e. the sink is the root of the spanning tree. The nodes which are in this range of the message of the sink, after receiving this message, first regulate the value of the variable of high LEVEL gateways with the sink and then activate the fuzzy module which is put in them to get a time value. When a node receives a value from its fuzzy module, it starts the countdown operation from the value get from the fuzzy module. While a node is counting down, if it receives a message from the neighboring node showing that another node has finished the countdown sooner, the mentioned node will stop the countdown and joins the other node.

When the node finishes the countdown (provided that it hasn't received the message of $\mathrm{CH}$-announce from the other available nodes in its radio rang, it will increase the value of its variable of LEVEL for one unit and disseminate a message of $\mathrm{CH}$-announce with the value of its current variable of LEVEL indicating that it has been determined as a $(\mathrm{CH})$. Each node chosen as $\mathrm{CH}$, selects itself as the LEVEL of the next stage. For example, when a node receives a message from the CH of LEVEL; 2, after the countdown and the dissemination of its message, introduces itself as the LEVEL; 3 . The value of the variable of LEVEL, corresponding with each $\mathrm{CH}$, is put in every message disseminated by it.

The nodes which receive the message of $\mathrm{CH}$-announce, while counting down, stop the countdown and set the value of the variable of the low LEVEL gateway with the ID of the node from which they have received the $\mathrm{CH}$-announce message. This work continues till the whole network is covered and then a tree-like is formed in the environment which can be called the backbone of data transferring to the sink. The following picture shows the form of the mentioned tree for a sample network.

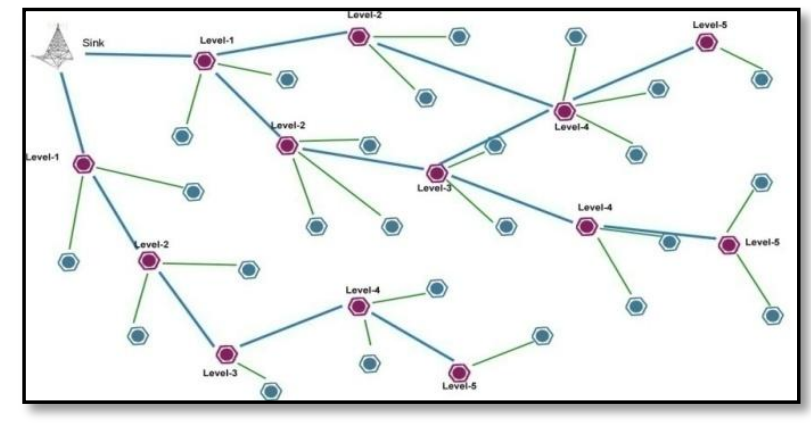

Figure 1. Network struct after setup phase

The red nodes are as $\mathrm{CH}$ and the blue ones as the member nodes. In the steady phase, the blue nodes join their corresponding red nodes and send their data to them. The $\mathrm{CH}$ nodes (red nodes) after the operation of data-aggregation on their received data from the member nodes, send their data to their previous level $\mathrm{CH}$ and send the $\mathrm{CH}$ of the previous level of the data to their proceeding level of $\mathrm{CH}$ and this job continuous till reaching the sink. The green lines are the route of transferring the member nodes' data to the corresponding $\mathrm{CH}$ and the blue lines are the route of transferring the data to the sink.

In the steady phase, those nodes which are not chosen as gateway node send their data to their corresponding gateway nodes (send their data to high LEVEL gateway). The gateway nodes after receiving their data do the operation of data aggregation and send them to their previous level (high LEVEL node). The previous LEVEL gateway to their previous LEVEL gateway and this continuous till they are sent to the zero level i.e. the sink.

Most of the clustering protocols first perform the setup phase and then the steady phase and after that the phases are continuously repeated along the running of the protocol. Our proposed plan has a main difference with these methods. When the setup phase ends, the network is covered by gateway nodes and the gateway nodes have different levels. In our proposed method the difference lies in that for every setup phase, two steady phases are run and then once more the setup phase is run. In other 
words, there is a setup phase after each two steady phases. In the odd rounds, the running of the member nodes protocol will join just the gateways with odd LEVEL and these gateways, after receiving the data will send them to the even LEVEL gateways to be sent to the gateways with lower LEVEL. That is to say, in the odd rounds, the even LEVEL gateways are used just to transfer the data of the odd LEVEL nodes among the environments. In the even rounds the operation is conversed i.e. the normal nodes join the gateway nodes with even LEVEL.

The following picture shows the way nodes join the gateway nodes in odd rounds. As it can be seen, in the current run (the odd round) the gateways with even LEVEL has no member and just transfer the data of the odd LEVEL.

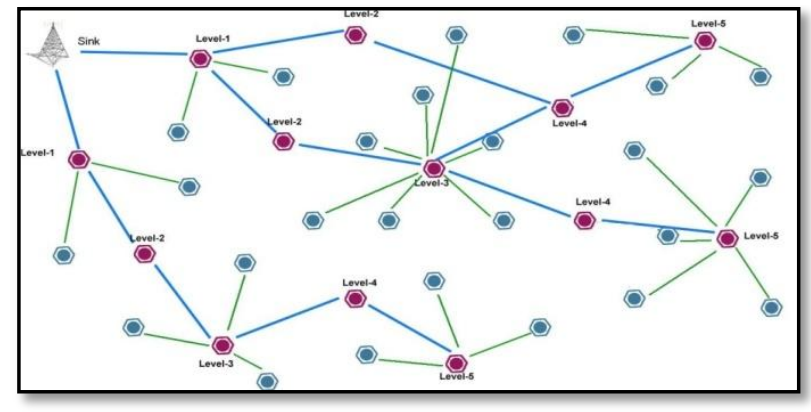

Figure 2. Network schema in odd round

The following picture (Figure 3) shows the way of network coverage in the even round which is opposite to the Figure 2.

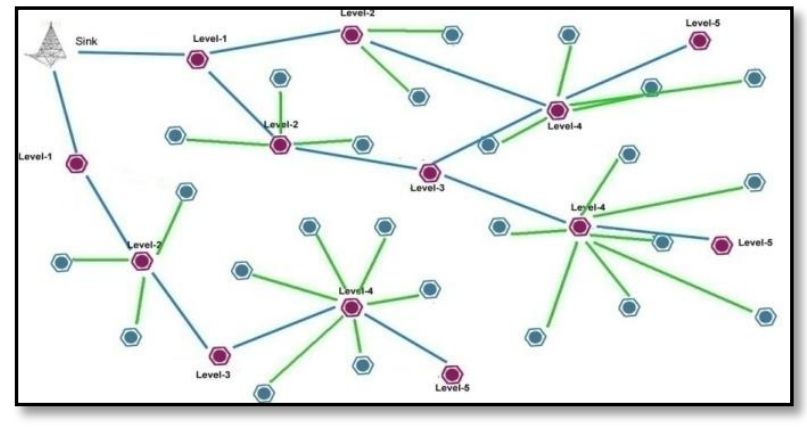

Figure 3. Network schema in even round

\section{Designing fuzzy module}

next issue to be dealt with is the fuzzy module which has to be implementation in each node. Considering some current parameters of each node in the setup phase, this fuzzy module obtains a number value for the countdown operation from which the related node starts counting down.

To design the fuzzy module, we have used three parameters as the entrances of the fuzzy module which are: the current energy of the node, the LEVEL of a node and the distance of the node from the node which it has received the $\mathrm{CH}$-announce message.

The reasons why these parameters have been used are as follows: the level of energy is the most basic parameter which has to be taken into account in the issue of the sensor networks and without this factor; the designing would be so inefficient. The more energy the node has, the more capable it is to be $\mathrm{CH}$ and vice versa.

The parameter of LEVEL has a direct relationship with the distance of a node from the sink. In the methods which the communication with the sink is through a single-hop, the nodes farther from the sink lose their energy sooner because the distance makes the energy drop much. But in multi-hop 
methods, it's quite conversed. It means that the nodes near the sink undergo much traffic i.e. they have to both transfer their own data and the data sent form the other nodes. That is because these nodes are the links to transfer the data to the sink. As a result, these nodes will stop working sooner.

Considering the aforementioned issues, the nodes having lower level, must have more energy. As our proposed method uses the multi-hop method, we have to take the second situation into account.

The last parameter i.e. the distance of the node from the node which it receives the $\mathrm{CH}$ message, suggests that when a node receives a message from its previous level indicating that it has become a cluster head, the node accepts it and wants to activate its fuzzy module to have the time value of the countdown. The node which is farther will be more appropriate, because the network can be covered with fewer gateway nodes with the same given range for the $\mathrm{CH}$-announce message.

The parameter of energy has language variables such as: v-low, low, med, high- v-high. The parameter of LEVEL too has the parameters of: low-med-high. And also the parameters of distance have the language variables of: near, med, far. The memberships function of each which is shown in the following:

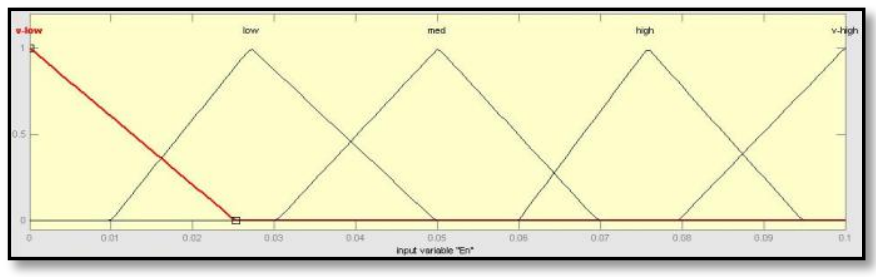

Figure 4. Energy membership function

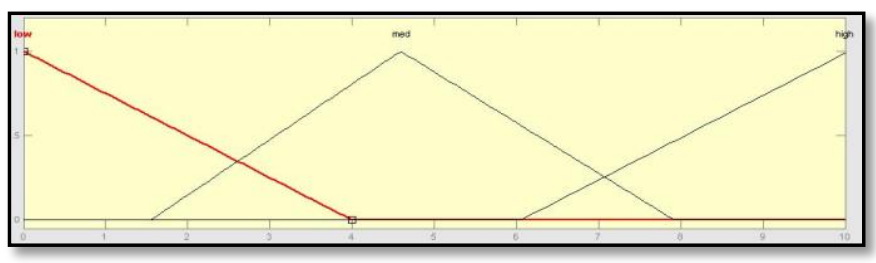

Figure 5. Level membership function

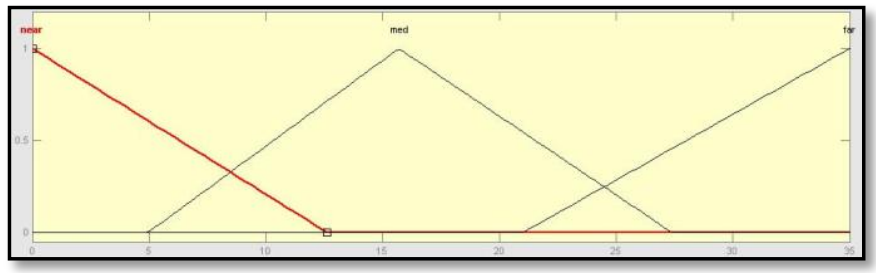

Figure 6. Distance membership function

The output of the fuzzy module is also shown in the picture below which get number value ranging from 0 to 30 for the countdown and its language variables are: v-short, short, med, long, v-long.

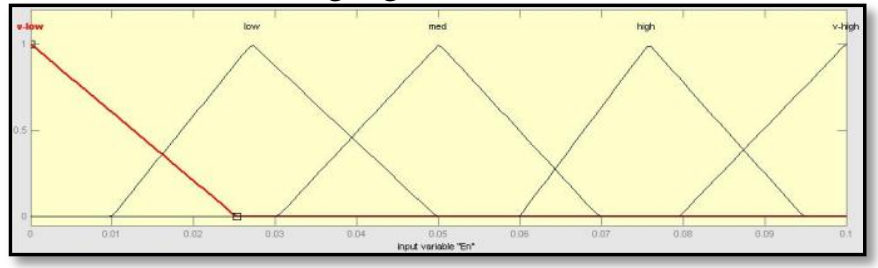

Figure 7. Output membership function 
For performing the following fuzzy operations, its corresponding operators have been chosen. The operator of And: min has been used The operator of Or: max has been used. For the defuzzification operation, the function of center of gravity has been used.

\section{Multi-sink}

The number of the sinks in the environment can be increased. The proposed protocol shows a good support for it. And there is no need to change the protocol at all. In the setup phase, each one of the sinks disseminates the $\mathrm{CH}$-announce message and starts covering the network. The only difference it has with the situation when there is just one sink in the environment is that in the single-sink situation, just one tree is formed its root which is the sink. But in multi-sink situation several covering tree with the root of sink get formed. In examining the results of the simulations, it will be shown that the increase in the number of the sinks improves the efficiency, because the number of the networks will be divided into smaller trees and the traffic pressure will be distributed among more nodes. As a result, the energy consumption will be distributed more.

\section{Supporting the mobile-sink}

In this section, to examine the mobile -hop, we will explain the way the mobile-hop is supported. We suppose that the sink is in the beginning of the protocol and at the edge of the network. Let's assume that the sink is at the edge of the network and the $\mathrm{CHs}$ have been created in the environment. The following picture (Figure 8) shows it. To make it simpler, the member nodes have been shown.

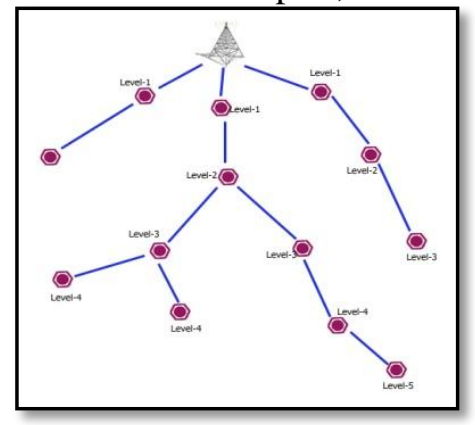

Figure 8. Network schema before sink change position

When the sink is at the edge of the network, the $\mathrm{CH}$ nodes will be on one side of the sink. As it can be seen, there are two nodes with LEVEL; 1 . They are the last nodes to transfer the other data of the CHs to the sink. Now if the sink wants to change its current position, first it sends a "s-ch-position" message to the LEVEL-1, indicating that it is changing its location. When the LEVEL-1 CHs receive this message they must store their data and other sent messages from the next level CHs in their buffer. After moving and fixing the position, the sink sends the "fixed-position" message, indicating that it has fixed its position this message has a specified range and there is certainly a $\mathrm{CH}$ in its range .when the message of the sink that shows it has fixed its position in the new location reaches a $\mathrm{CH}$, the related $\mathrm{CH}$ sends a message indicating the creation of a new route to the sink which has the $\mathrm{CHs}$ with lower (high) LEVEL than itself and this message goes to the root of the sub tree which this $\mathrm{CH}$ is a member of (in other words it goes to the LEVEL-1 node at the tree). When the LEVEL-1 CH receives this message, it disseminates a message twice as big as the radius of the $\mathrm{CHs}$ in order for the neighboring level1 $\mathrm{CH}$ to know that the new position of the sink is in the low levels of the tree which this node is a root of. The following picture (Figure 9 to Figure 11) shows the mentioned stages. 


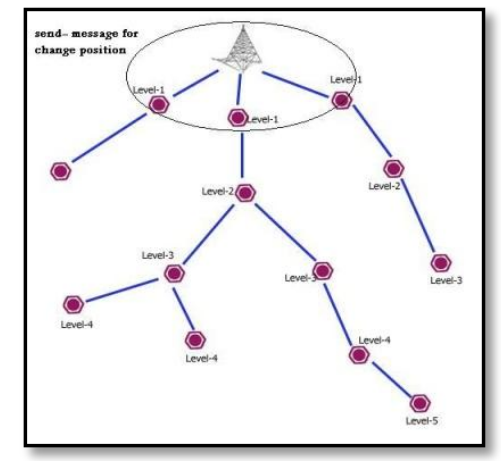

Figure 9. Send "s-ch-position" message from sink for gateway of LEVEL-1

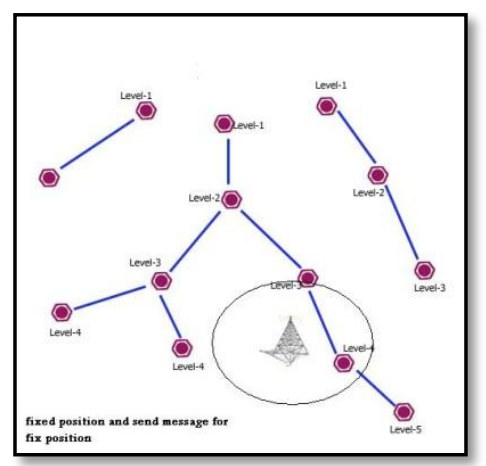

Figure 10. Sink send "fixed -position" message that fixed

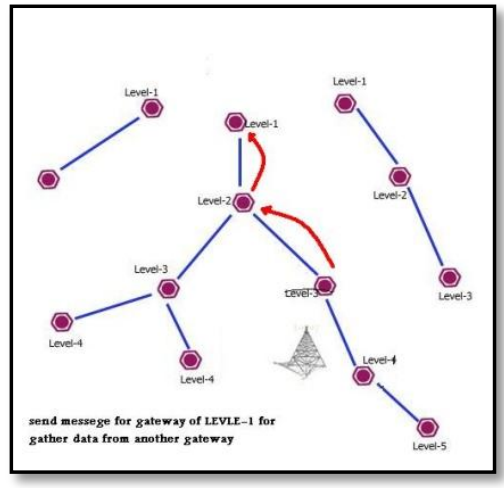

Figure 11. Exchange message between gateways to message reach to gateway of LEVEL-1

Now the LEVEL-1 CH which the sink is not at its lower levels receives the data related to its lower level $\mathrm{CH}$ and sends those data to the LEVEL-1 $\mathrm{CH}$ from which it has received the previous level message and the node sends the data to the upper level $\mathrm{CH}$ in which the sink has announced its position. In fact, the number of all the control packets which are moved equals the maximum level of the tree. That is to say, if $\underline{\mathrm{d}}$ is the deepest sub tree available, we at most need the $\underline{\mathrm{d}}$ of control packet for the proper moving of the messages of the nodes to the sink.

The mentioned material has been shown below. First the green color $\mathrm{CH}$ sends itself to the red color $\mathrm{CH}$ because the sink lies in its sub-tree and then this $\mathrm{CH}$ will send the data to the opposite direction to which it has received the fixing message of the sink. 


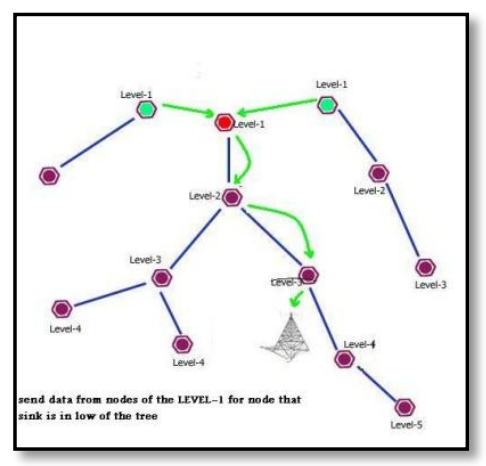

Figure 12. Now another gateway of the LEVEL-1 sends data for gateway specify

In the preceding picture, the level $2 \mathrm{CH}$ which the sink is at its lower level, does not send the received data from the level $3 \mathrm{CH}$ of its right hand sub-tree to the level $1 \mathrm{CH}$; rather pushes it to the $\mathrm{CH}$ which is related to the sink. It must be noted that, this data transfer situation is performed just in the current round and in the next phase of the setup, the sink is covered again from the new position of the sink and there is no need to transfer such data.

\section{The simulation results}

The simulation has been done through matlab and the results of the proposed method have been compared with LEACH clustering methods, HEED, the fuzzy method presented in CHEEF and the method presented in the article of RCSDN. In the simulation environment, the environment parameters have been taken into account as the table 1 show:

TABLE I. SIMULATION PARAMETERS

\begin{tabular}{|l|l|}
\hline \multicolumn{1}{|c|}{ Parameter } & \multicolumn{1}{c|}{ Value } \\
\hline Network size & $100 * 100 \mathrm{~m}$ \\
\hline Base station location & $50,50 \mathrm{~m}$ \\
\hline Initial energy for node & $0.1 \mathrm{Joule}$ \\
\hline$E_{\text {elec }}$ & $50 \mathrm{~nJ} / \mathrm{bit}$ \\
\hline$\varepsilon_{f s}$ & $10 \mathrm{pj} / \mathrm{bit} / \mathrm{m} 2$ \\
\hline$\varepsilon_{m p}$ & $0.0013 \mathrm{pj} / \mathrm{bit} / \mathrm{m} 4$ \\
\hline Data aggregation energy & $5 \mathrm{nj} / \mathrm{bit} / \mathrm{signal}$ \\
\hline$d_{0}$ & $87 \mathrm{~m}$ \\
\hline
\end{tabular}

\subsection{The life time of the network and other parameters}

The primary amount of the node's energy has been taken as: 0.1 joule. Based on the following table (Table 2.), the results from the simulation for a network with 100 square meter area in 100 square meter area has been obtained.

TABLE II. MOBILE SINK SIMULATION RESULT AND ANOTHER PARAMETERS
\begin{tabular}{|l|c|c|c|c|c|}
\hline \multicolumn{1}{|c|}{ paramerts } & LEACH & HEED & CHEEF & RCSDN & MHRMS \\
\hline First-dead & 106 & 115 & 120 & 123 & 168 \\
\hline $\begin{array}{l}\text { Mean count control } \\
\text { packet }\end{array}$ & 10 & 18 & 13 & 21 & 19 \\
\hline $\begin{array}{l}\text { Not covered to } \\
\text { first-dead }\end{array}$ & 0 & 0 & 0 & 0 & 5 \\
\hline
\end{tabular}

Now we compare the results of the shown algorithm in different forms with various numbers of nodes and the network area for the first-dead and result show in Table 3.

TABLE III. FISRT-DEAD SIMUALTION RESULT FOR NETWORK WITH DIFFERENT NODE AND SIZE 


\begin{tabular}{|l|l|l|l|l|l|}
\hline \multicolumn{1}{|r|}{ Algorithm type } & & & & \\
$\begin{array}{l}\text { Network size and node } \\
\text { count }\end{array}$ & 97 & 110 & 105 & 108 & 133 \\
\hline $\begin{array}{l}150 \text { node in Network size } \\
150 * 150\end{array}$ & 82 & 103 & 96 & 100 & 115 \\
\hline $\begin{array}{l}200 \text { node with Network size } \\
200 * 200\end{array}$ & 69 & 84 & 83 & 78 & 108 \\
\hline $\begin{array}{l}300 \text { node with Network size } \\
300 * 300\end{array}$ & 51 & 69 & 70 & 63 & 81 \\
\hline $\begin{array}{l}400 \text { node with Network size } \\
400 * 400\end{array}$ & 50 & & & \\
\hline
\end{tabular}

\subsection{The effect of the sink position on efficiency}

Now, we have changed the condition of running the algorithm and have measured the effect of the sink position on the efficiency of the algorithm. It means that we have put the sink in different positions and have assessed the efficiency of the algorithms. The result of the simulation and running the algorithm from the first-dead to happen are as follows (Table 4.): the number of the nodes is 100 and the network is $100 \times 100$ square meter.

\begin{tabular}{|c|c|c|c|c|c|}
\hline Algorithm type & $\frac{2}{3}$ & 武 & 愛 & $\hat{\Omega}_{2}^{\pi}$ & $\underset{\mathbf{z}}{\mathbf{3}}$ \\
\hline $\begin{array}{l}\text { Sink is in center of network } \\
\text { environment }\end{array}$ & 100 & 102 & 95 & 96 & 133 \\
\hline $\begin{array}{l}\text { Sink is in border of network } \\
\text { environment }\end{array}$ & 65 & 78 & 72 & 75 & 115 \\
\hline $\begin{array}{l}\text { Sink is } 100 \mathrm{~m} \text { far from border of } \\
\text { network environment }\end{array}$ & 13 & 49 & 51 & 59 & 95 \\
\hline $\begin{array}{l}\text { Sink is } 200 \mathrm{~m} \text { far from border of } \\
\text { network environment }\end{array}$ & 3 & 26 & 13 & 10 & 64 \\
\hline
\end{tabular}

In the methods such as: LEACH and CHEEF which use single-hop method to send the data to the sink, the farther the sink is from the sensor environment, the more decrease we will see in the efficiency of the algorithm. This is so because the nodes as $\mathrm{CH}$ which are in charge of transferring the data, have to transfer them longer distance. As it can be seen in the table, when the distance goes beyond the threshold limit, the nodes using the single-hop method will lose the energy of the nodes quickly. Because in this condition the energy consumption is related to power 4 of the distance.

\subsection{The number of the cluster-heads and the average distance of the data dissemination} In the following picture, we have compared the number of the cluster heads until the time of the first dead in the network between the proposed method and the method of LEACH. 


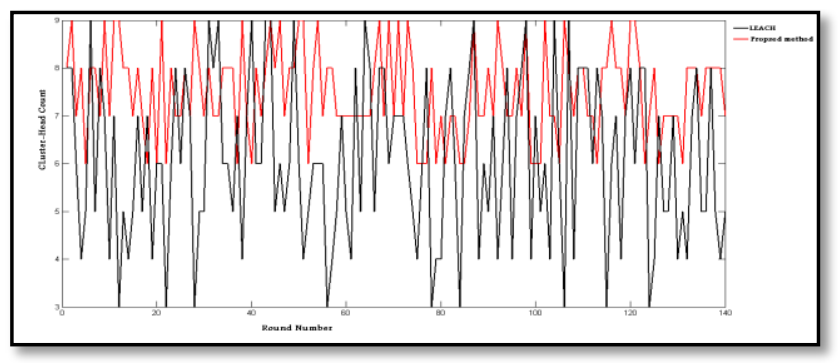

Figure 13. Cluster-head count to first-dead for LEACH and MHRMS

The red color graph shows the number of the cluster heads in each round in the proposed method and the black color one shows the number of the cluster heads in the LEACH method. As it can be seen, in the LEACH method the number of the clusters in different rounds has high variance and as a result, in many of the rounds we are far from the optimal condition. The following graph shows the average distance to which a node must disseminates its data. In this graph the sink is on the upper edge of the network and the proposed method of the average distance of dissemination in each round has a close relationship with the cluster radius and is so close to it. But in the methods such as LEACH, this high distance will have a striking difference in each round. In the following picture, the red color graph shows our proposed plan and the black color one shows the LEACH method. (In the simulation, the radius of the cluster is taken 30 meters).

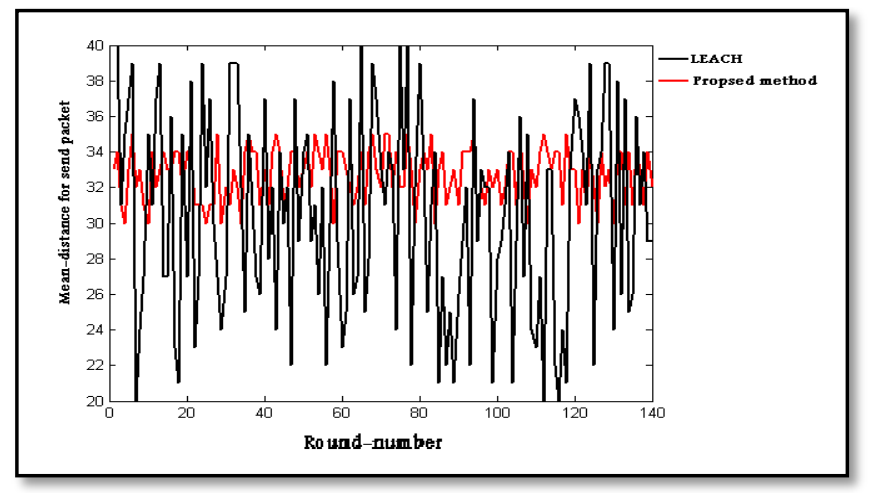

Figure 14. Mean distance for send packet in $\mathrm{CH}$ nodes

\subsection{Multi-sink}

We have also examined our proposed method in an environment where there are more than one sink. In this situation there are two sinks in both sides of the environment. The result of simulation when there are two sinks in the network is shown below.

TABLE V. FIRST-DEAD SIMULATION RESULT FOR SINGLE-SINK VS MULTI-SINK
\begin{tabular}{|c|l|c|}
\hline Network size and node number & Single-sink & Multi- sink \\
\hline 150 node in Network size $150 * 150$ & 133 & 158 \\
\hline 200 node with Network size $200 * 200$ & 115 & 127 \\
\hline 300 node with Network size $300 * 300$ & 108 & 115 \\
\hline 400 node with Network size $400 * 400$ & 81 & 100 \\
\hline
\end{tabular}

As it was mentioned, when there are more than one sink in the environment, the pressure of the duty of transferring the data of the other $\mathrm{CHs}$ will get divided into two groups of level-1 cluster heads. As a result the energy consumption of these nodes decreases and their life span will increase. Moreover, the number of moved control packets is less and the growth of the level of relay will be less and the network will be divided into two smaller sub trees. The following picture shows the number of created levels. As it can be seen, the number of levels in multi-sink method is almost half the number in a single - sink method. 


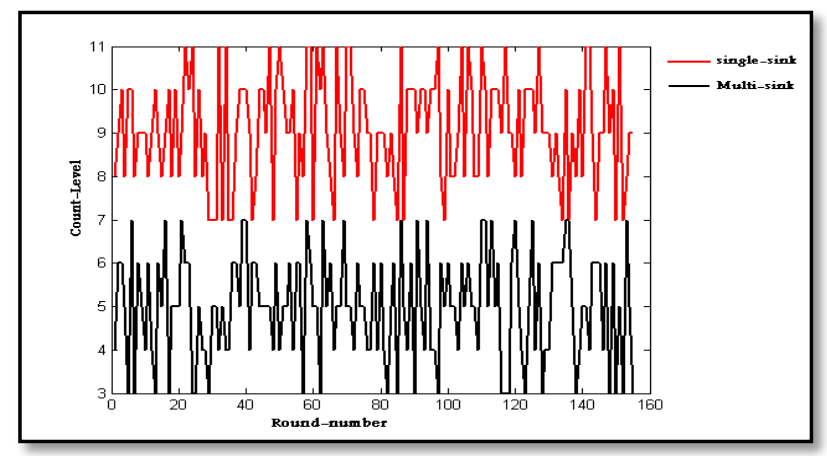

Figure 15. Count level created between single-sink with multi-sink

\subsection{Examining the efficiency of mobile-sink method}

We have supposed that there is a network with 200 nodes with the dimensions of $200 \times 200$ and the sink at first is in the middle of the edge which is up the environment. In each stage the sink changes its position with the probability of 0.3 and does not change its current position with the probability of 0.7 . The table below shows the results of running the algorithm.

\begin{tabular}{|l|c|}
\multicolumn{1}{|c|}{ TABLE VI. MOBILE SINK SIMULATION RESULT AND ANOTHER PARAMETERS } \\
\begin{tabular}{|l|c|}
\hline \multicolumn{1}{|c|}{ Parameter } & Values \\
\hline First-dead & 109 \\
\hline Deep created tree & 6 \\
\hline Count change sink position & 43 \\
\hline Mean count control-packet for change sink position & 3 \\
\hline Mean decrease energy for each round & 0.0035 \\
\hline
\end{tabular}
\end{tabular}

As it is shown in the above table, the difference of energy consumption and other parameters when the sink is fixed in the environment is not much. And it shows that the efficiency in the mobile-hop has been preserved.

\section{Conclusions}

In this paper a multi tracking fuzzy method with the capability of supporting the sink has been introduced. The fuzzy logic has been used for choosing a node and as a relay to transfer the data to the sink. The main feature of this method is that the increase in the number of the nodes and sinks does not have an undesirable effect on the efficiency of the network and as a result this protocol enjoys a good measurability. Furthermore the results of simulation showed that it is possible to support the mobile hop in this protocol and using fewer extra control packets than the common methods, we can have this possibility in the protocol. The results of simulation with other methods too, show the efficiency of this method

\section{References}

[1] S.-C. Liu, "A lifetime-extending deployment strategy for multi-hop wireless sensor networks," in Proc. CNSR, May 2006, pp. 53-60.

[2] Wireless Sensor Network Designs. Hoboken, NJ: Wiley, 2003.

[3] T. S. Chen, C. Y. Chang, and J. P. Sheu, "Efficient path based multicast in wormhole-routed mesh networks," J. Syst. Archit., vol. 46, no. 10, pp. 919-930, Aug. 2000.

[4] M. Younis, P. Munshi, G. Gupta and S. M. Elsharkawy, "On Efficient Clustering of Wireless Sensor Networks", Second IEEE Workshop on Dependability and Security in Sensor Networks and Systems, 2006, pp.78-91.

[5] W.R. Heizelman, A. Chandrakasan and H. Balakrishnan, "Energy-Efficient Communication Protocol for Wireless Micro Sensor Networks",IEEE Proceedings of the Hawaii International Conference on System Sciences, Jan 2000. 
[6] M. Chatterjee, S.K. Das and D. Turgut, "WCA: a Weighted Clustering Algorithm for mobile Ad Hoc networks", Journal of Cluster Computing (Special Issue on Mobile Ad hoc Networks), vol. 5, no. 2, pp. 193204,April 2002.

[7] A.G. Delavar, J. Artin and M. M. Tajari," RCSDN : a Distributed Balanced Routing Algorithm with Optimized Cluster Distribution", IN 3rd International Conference on Signal Acquisition and Processing (ICSAP 2011)

[8] I. Gupta, D. Riordan, and S. Sampalli, "Cluster-head election using fuzzy logic for wireless sensor networks," in Communication Networks and Services Research Conference, 2005. Proceedings of the 3rd Annual, 2005, pp. 255-260.

[9] J. Kim, S. Park, Y. Han, and T. Chung, "CHEF: cluster head election mechanism using fuzzy logic in wireless sensor networks," in Proceedings of the ICACT, 2008, pp. 654-65.

[10] 0. Younis and S. Fahmy, "Distributed Clustering in Ad Hoc Sensor Networks: A Hybrid, Energy-Efficient Approach," Proc. IEEE INFOCOM, Hong Kong, Mar. 2004; an extended version appeared in IEEE Trans. Mobile Comp.,vol. 3, no. 4, Oct.-Dec. 2004, pp. 366-79.

[11] C. Intanagonwiwat, R. Govindan, and D. Estrin, "Directed Diffusion: A Scalable and Robust Communication Paradigm for Sensor Networks,” Proc. ACM MobiCom '00, pp. 56-67, Aug. 2000.

[12] M. Chen, T. Kwon, and Y. Choi, "Energy-Efficient Differentiated Directed Diffusion (EDDD) in Wireless Sensor Networks," Computer Comm., vol. 29, no. 2, pp. 231-245, Jan. 2006.

[13] F. Ye, H. Luo, J. Cheng, S. Lu, and L. Zhang, "A Two-Tier Data Dissemination Model for Large-Scale Wireless Sensor Networks,” Proc. ACM MobiCom '02, Sept. 2002.

[14] B. Zhang and H. Mouftah, "Energy-Aware on-Demand Routing Protocols for Wireless Ad Hoc Networks," Wireless Networks, vol. 12, no. 4i, pp. 481-494, Aug. 2006.

[15] Z. Liu, D. Xiu, and W. Guo, “An Energy-Balanced Model for Data Transmission in Sensor Networks,” Proc. 62nd IEEE Vehicular Technology Conf. (VTC-Fall), 2005.

[16] A. Boukerche, I. Chatzigiannakis, and S. Nikoletseas, "A New Energy Efficient and Fault-Tolerant Protocol for Data Propagation in Smart Dust Networks Using Varying Transmission Range,” Computer Comm., vol. 29, no. 4, pp. 477-489, Feb. 2006.

[17] I. Haque and C. Assi, “OLEAR: Optimal Localized Energy Aware Routing in Mobile Ad Hoc Networks," Proc. IEEE Int'l Conf. Comm. (ICC '06), pp. 3548-3553, June 2006.

[18] S. Wu and K. Candan, "Power-Aware Single- and Multipath Geographic Routing in Sensor Networks," Ad Hoc Networks, vol. 5, no. 7, pp. 974-997, Sept. 2007.

[19] N. Wang and C. Chang, "Probabilistic-Based Energy Aware Routing for Network Lifetime and Source Level Fairness in Wireless Sensor Networks," Proc. Int'l Conf. Systems and Networks Comm. (ICSNC), 2006. 